\title{
Comparing the Similarities and Differences between All Versions of Grand Theft Auto
}

\author{
Cagri Baris Kasap \\ Istanbul Gelisim University, Istanbul, Turkey \\ Email: cagribkasap@hotmail.com
}

How to cite this paper: Kasap, C.B. (2018) Comparing the Similarities and Differences between All Versions of Grand Theft Auto. Journal of Software Engineering and Applications, 11, 89-109.

https://doi.org/10.4236/jsea.2018.112005

Received: December 25, 2017

Accepted: February 10, 2018

Published: February 13, 2018

Copyright $\odot 2018$ by author and Scientific Research Publishing Inc. This work is licensed under the Creative Commons Attribution International License (CC BY 4.0).

http://creativecommons.org/licenses/by/4.0/ (c) (i) Open Access

\begin{abstract}
The article investigates the similarities and differences between all versions of Grand Theft Auto as an adventure game with the widest popularity in the last decade. The game is a story collection, a frame for performance, a virtual museum of vernacular culture and a widely circulated pop culture artifact whose double-voiced aesthetic has given rise to diverse interpretive communities. The aim of comparing the differences and similarities between different versions of the game is to be able to evaluate the game from the user's point of view. With this aim, whether with the verisimilitude that the different versions offer makes GTA a product of an iterative design process or not will be displayed.
\end{abstract}

\section{Keywords}

Grand Theft Auto (GTA), Iterative Design, Adventure Game, Vernacular Culture

\section{Introduction}

Grand Theft Auto has first been composed by David Jones and Mike Dailly some time ago by what was then-known as DMA Design Company (in view of its spearheading power, this studio was one of the astounding names in the field), which has later on been changed over to Rockstar North. The game was distributed by Rockstar Games.

Electronic games, with their mechanical roots in the military-industrial complex, are for the most part associated with childish play and lowbrow excitement for the antisocial-all of which hurts their legitimacy as things of certifiable social idea [1]. Exactly when savants of savage electronic games see Grand Theft Auto, they see a stunning transport contraption for the fight-prepared culture of fierceness, one that targets the powerless identities of children. Furthermore, to 
the extent that the gut factor, uncommon foulness, and the sheer chafe of game designers, there is likely nothing spoilers could state concerning the redirection that would not be certifiable. Grand Theft Auto is a draining confusion, and planners have clearly drawn on Hollywood orders of extraordinary slasher films, swarm experiences, auto chases, and a rich legacy of action ventures. Presented is a movement of American trivialities, packaged, promoted, and sold back to us. The delight gives off an impression of being an adjustment of social talk and shrewd social aftereffect of combining America's cutthroat industrialist inspiration with a valorized national legacy of ruthlessness and hegemony.

Regardless, what is more intriguing than the moral freedom all around the depiction of ruthlessness is the methods by which GTA, through a reproduced "reasonable" feeling of space and time, passes on a sweeping sentiment "place". That is to express, a player's ability to act inside a gaming space is made unmistakable through the viable mix of picture, physicality, and sound. By making sense of how to sufficiently investigate a reenacted body inside this appearance, the idea of place springs up. As needs be, it addresses a persuading human-PC encounter between edifying space and lived space. Likewise, it is through unscripted, performed, free-play in this significantly verbalized place that the solid social examination shrouded Grand Theft Auto snaps distinctly into focus.

As it is understood, the guideline point of the game is car robbery. At first happening in rural London, the setting of the redirection is accessible day world with scenes from whimsical American urban zones involving a blend of New York City, Vice City, Miami, California and Nevada.

A wannabe or a criminal is the basic character and he needs to climb through the ranks of organized crime. The player is set in the position of this criminal and is given distinctive missions by managers and other genuine symbols that ought to be done all through the story structure. Assassinations and distinctive crimes related to car-stealing, firefighting, street dashing and seizing helicopters and settled wing flying machine include the essential activity enterprises of the game.

The Grand Theft Auto has a place with a sort of free-wandering games called sandbox games and gives much measure of adaptability to the player. The larger part of the games has a place with the structure of single-track course of action with straight gameplay. Grand Theft Auto allows the player to choose the missions he needs to endeavor so the relationships with various characters in the storyline are changed too. The urban regions in the game describe the redirection in the open world class in which open structures with minor missions inside the storyline are publicized.

Iterative design is a design system in light of a cyclic procedure of prototyping, testing, analyzing, and refining a product or process. In light of the aftereffects of testing the latest cycle of a design, changes and refinements are made. In this light, looking at all the forms of Grand Theft Auto, it will be made sense of whether creators of GTA have inferred an iterative design or not.

One of the fundamental reasons iterative design is essential is that it enables 
groups to diminish ease of usability issues and consequently guarantee a decent user experience of the product they are creating. Then again, designers can uncover these defects at the beginning stages when the cost of killing slip-ups is insignificant.

\section{Version 1-Grand Theft Auto (1997)}

As the main variant of the game, it is set in three distinctive anecdotal urban areas, Liberty City, San Andreas and Vice City. A lessened Game Boy Color port of this adaptation has additionally been discharged later on.

The main adaptation is comprised of a progression of levels, every one set in one noteworthy city. The player's goal is to perform errands for the city's neighborhood crime syndicate so a specific measure of focuses is accomplished. Fruition of a mission by expanding the player's "multiplier" opens the chance to endeavor for higher prizes while disappointment grants few focuses and decrease open doors for accomplishing more assignments. Dissimilar to later games in the series, in the primary form, the player can be squandered in one hit without body protection. On the off chance that the player gets squandered, he loses an existence. On the off chance that the player is squandered too often, he/she begins the level starting with no outside help.

A standout amongst the most vital highlights of Grand Theft Auto is the flexibility in the decision that it gives to the player. In spite of the fact that the goal is typically settled, amid the mission, there is still some flexibility to pick the course to take. It was this level of flexibility that isolated GTA from different games at the time. The player is chiefly free in doing whatever he needs to do. The player can pick up focuses by causing devastation in the midst of the movement or take or offer autos for benefit. To develop the multiplier, the player will finish in any event a few missions to get the cash required to finish a level. Some criminal demonstrations have inalienable multiplier i.e. utilizing a squad car for running over individuals pairs the points received.

Beginning from the 1997 version, the technique of Ambient Occlusion has been utilized [2]. Ambient Occlusion ( $\mathrm{AO}$ ) includes contact shadows where two surfaces or objects meet, and where a question squares light from achieving another close-by game component. In Grand Theft Auto V however, the setting's slightly bugged, and is anticipating a fix to reestablish full usefulness. In the wild, Ambient Occlusion applies proper levels of murkiness to shadows covering foliage and guarantees foliage that falls under a shadow is accurately shadowed. In urban territories, we watch a large increment in devotion around the structures in the frontal area and some unpretentious changes out of sight. In its current, to some degree practical state, Ambient Occlusion costs a couple of edges for every second and adds enormously to picture quality making it an absolutely necessary setting.

Another strategy that is utilized beginning from this form is Anisotropic filtering [2]. Anisotropic separating sharpens far off surfaces and those viewed on 
an edge. In Grand Theft Auto V, it functions as you would expect and with an insignificant execution affect.

\section{Version 2-Grand Theft Auto II (1999)}

As the main variant with numbered continuation rather than a Roman numeral, Grand Theft Auto 2 was the second arrangement of the game. It was created for Microsoft Windows, PlayStation, and Dreamcast. It was discharged in the year 1999 and was set in an indeterminable, retro-advanced anonymous American city as its setting and highlighted refreshed illustrations and to some degree distinctive gameplay in light of the player's interest to different criminal associations. It is the main form that had a " $\mathrm{T}$ " rating for Playstation support, be that as it may, its Game Boy Color port was likewise created.

With Grand Theft Auto 2, the storyline is all the more unfathomably created. The player is compelled to defeat an awful occasion that will lead the character to the high grounds of the stepping stool of sorted out crimes and prompts his triumph. GTA2 held the overhead perspective of the GTA and additionally the car-stealing/phone-replying recipes of the main version. The player has greater capacity to investigate urban communities by walking or in vehicles with the point of accomplishing a specific score. Simply after a specific score, can the player move to the following level. Doing the missions grants the player points yet they are not basic for the finishing of the game.

One element that stood unique in relation to the primary form was the capacity to do missions for isolated posses in the meantime. With the way that completing one mission for one gang would decrease the level of push of another, the distinctive is that SWAT groups are presented in the downtown locale while Special Agents and Army officers are experienced in the private and modern areas. The separating kinds of law implementation start pursuing the player all the more effectively as the level increments.

The city action as a rule expands. Passing pedestrians and vehicles are not any more simply corrective appearances however in some cases walkers would periodically ride taxicabs or transports. Pedestrians, group individuals and police and in addition carjackers and muggers would take part in battles in a similar city.

What could be depicted as "side-missions", for example, being a cab driver or a semi-truck driver with shrouded bundles and a wellbeing meter are in play too. Being a cab driver would win you one dollar for every second. When stopping, travelers could get out on the off chance that they need to, making the motors stop.

A few weapons highlight a "Kill Frenzy" mode. At the point when taken up, a player has a constrained measure of time to kill certain number of individuals with it. Or on the other hand if a player finds a tank and gets in it, a "Kill Frenzy" mission begins. In arbitrary parts of the city exceptional autos are stopped and if the player gets on one of them, he needs to murder individuals 
either by running over them or with the additional weapon that the car has.

The Playstation version of Grand Theft Auto 2 is conditioned down from the $\mathrm{PC}$ adaptation with bring down portions for the quantity of executes required in frenzy style missions [3]. The port additionally incorporates an element where the player car will detonate after the player executes countless individuals. Rather than the player deceiving regular folks into entering a transport to drive to a meat handling plant to be torn up, the casualties are posse individuals from Hare Krishna.

There has been a motion picture, "GTA2: The Movie" that has been discharged in 1999. The film's setting was in New York City rather than the mysterious city without bounds that the diversion was occurring in. The film was produced out of an eight-minute short film that has been made to advertise the game.

Another technique that has started to be utilized beginning from this version is Extended Distance Scaling [2]. It is a technique that was a move up to Distance Scaling that was utilized as a part of the past version. It helps render additional detail in Distance Scaling's sphere of impact and additional detail at long range past said circle. This is particularly clear in this arrangement of comparisons, where each and every game component is overhauled with extra detail and new detail is rendered on the slope underneath the Vinewood sign.

Of all systems, Extended Distance Scaling has the largest execution affect when you factor in the additional concurrent hit from civilians, substantial activity, police pursues, blasts and grass which is difficult to precisely depict in benchmarks.

Another strategy that is utilized is Extended Shadow Distance [2]. Notwithstanding expanding the range at which shadows are rendered, Extended Shadow Distance builds the detail level of shadows, includes extra shadows at all separations and builds the precision of shadows, changing over a guestimated foggy shadow into a precisely rendered, point by point shadow that is effectively shown.

\section{Version 3-Grand Theft Auto III (2001)}

After a whole deal and a straight-forward discussion about the eventual fate of the game, with setting in anecdotal Liberty City, freely like New York City with components from other American urban areas, Grand Theft Auto III was discharged in October 2001, and filled in as the achievement for the establishment. After the discharge of GTA III, the game was said to return back to the gaming market.

What was earth shattering was the third-individual view (instead of the camera-points in which the past variants were clutched) that held in view a little guide that enabled the user to trigger in the sandbox (open world) game for route. With its third-individual shooter and driving game in a game engine environment with the multiplying of DMA Design of Body Harvest being the first 
endeavor to have a 3D game engine in such a genre.

Criminal offenses like carjacking, murder and burglary will bring about expanding levels of protection from the authorities. On the off chance that the player turns out to be more "needed", the police, FBI or armed forces react. At the point when the player falls from his wounds or is captured, he will be taken to a neighborhood healing facility or police headquarters and will lose his whole cash as renumeration for the therapeutic costs. While this is like the past Grand Theft Auto and Grand Theft Auto 2, the player has basically boundless lives that permit him/her to kick the bucket as many number of times as s/he wants and vastly lose in the game.

Rather than the negligible crime commitment in the past versions, in GTA III, it is just auto smashing, vehicle obliteration or passerby slaughtering that permits the player to get money. What is new is additionally that the measure of cash does not enable the player to open new regions however as new territories open up past regions turn out to be more perilous.

The interface is unique in relation to the past versions too. The compass has been changed by a smaller than expected guide that additionally shows a guide of the city's key spots and areas. Protective layer and wellbeing levels are shown in numbers and a 24-hour clock is included. Group conduct is never again directed by the regard level however rather as the player's advance all through the storyline influences his view in the group individuals' eyes. As the player finishes the missions for various posses, gang members will recognize the character.

The multiplayer mode that enabled players to interface through a system and play with each other was brought down to a solitary player alternatives in GTA III. To lessen the gap of the lacking players' system, outsider changes, for example, Multi-Robbery Auto was produced and discharged for future successors.

Different augmentations were produced in a more mission-situated approach in plot and in addition new levels in voice-acting. This version likewise offers radio stations which recreate heading to music with plate racers, radio identities, ads, talk radio and American Pop Culture.

By October 2011, the iPad, iPhone, Android tablet arrivals of the adaptation was reported keeping in mind the end goal to praise the game's tenth year significant discharge.

Another strategy that is utilized beginning from this version is High-DetailStreaming While Flying [2]. High-Detail-Streaming While Flying goes about as a Level of Detail setting when the player is uncertain, diminishing visual constancy to enhance execution. This likewise expands pop-in, however it is significant that even with each amusement setting at most extreme, pop-in while flying is unavoidable. Despite the fact that with this strategy, it is much more discernible. In the in-amusement benchmark, performance contrasts by a little more than four edges for each second. Amid gameplay in the city, be that as it may, the effect has all the earmarks of being higher.

The last technique that is utilized as a part of this version is High-Resolution 
Shadows [2]. High-Resolution Shadows increase the detail level of shadows. In any case, this is commonly observable just when utilizing "Sharp" Soft Shadows, rather than one of the other delicate choices. The case underneath likewise exhibits how the detail of shadows changes relying upon the separation from the shadow caster, the remove from the player's purpose of and the surface the shadow is given occasion to feel qualms about.

This propelled shadow setting guarantees most extreme shadow loyalty however with its moderately extensive performance affect players on lower-end frameworks advantage more from empowering "Soft" soft shadows, rather than "Sharp" High Resolution Shadows. This will cover the loss of shadow loyalty as well as the expansion in shadow associating when High Resolution Shadows are crippled.

\section{Version 4-Grand Theft Auto-Vice City (2002)}

This is the form in which the game was changed into an open-world activity experience computer game. The designs utilized were 3D-arranged conditions of Vice City, Miami as the main setting in which this particular form was outlined to happen.

The game has a comparable gameplay outline and interface with GTA III. The gameplay is open-ended to the extent that missions are finished to open the obscure parts of the city. In the meanwhile, the player can drive to the diverse parts of the city and do whatever s/he needs to do similar to $s /$ he is not in the middle of a mission.

Player can take vehicles (even helicopters) and share in shootings and thefts so as to make mayhem. Nonetheless, doing as such creates deadly consideration from the police (or FBI, National Guard). In spite of the fact that police conduct can be killed in a few ways, it is like the past form aside from that officers can employ nightsticks and spike strips to cut the feels worn out on the player's car

Another extra component is the capacity to purchase various properties dispersed over the city [4]. There are additionally a dance club, a strip club, a taxi organization, ice-cream conveyance organization, a boatyard, a printing acts and additionally an auto showroom that the player can purchase as resources. Every business property has various missions connected to it and once every one of the missions are finished, the property will start to produce a progressing wage.

The event of groups is not quite the same as the previous adaptations. Packs that are basic to the story occasions show up in the game. These groups ordinarily have a positive or negative conclusion of the player and either take after the player or shoot at him. There is a continuous battle between the groups and a few missions saved for the player include sorting out battles between contradicting groups.

Like the previous adaptations, discretionary side-missions are composed. They incorporate making pizza conveyances, driving harmed individuals to a doctor's facility with an emergency vehicle, dousing fires with a fire truck, con- 
veying travelers in a taxi, utilizing a police vehicle to slaughter culprits and driving a transport with toll paying travelers. There are financial honors and also gameplay points of interest connected to these missions.

This version was produced by RockStar North in United Kingdom and distributed by RockStar Games. It has appeared in North America on 29 October 2002 for Playstation 2 and was later ported to Xbox and Microsoft Windows one year afterward. A Nintendo Game Cube adaptation was arranged however never has been discharged.

One technique that was utilized with this adaptation was In-Game Depth of Field Effects [2]. Accessible when PostFX is set to Very High or Ultra, Depth of Field is an individual inclination procedure, much the same as Motion Blur. Impartially, it helps frame cutscenes and concentrate the camera on a particular occasion or area however once in a while, it can incorrectly obscure the whole screen amid gameplay, before jostling snapping back to a sharp picture.

Another strategy that was utilized with this adaptation was that of Long Shadows [2]. The Long Shadows choice renders more precise shadows amid the game's sunset and first light eras (physically accessible by means of Director Mode). The impact is subtly however that couple of players will accurately recognize the impact's utilization in a one-next-to-the-other on-off test.

\section{Version 5-Grand Theft Auto-San Andreas' (2004)}

This version is intended to occur in San Andreas, Los Angeles as its setting. It was produced by RockStar North and distributed by RockStar Games. Initially discharged for the Playstation 2 of every 2004, the game was discharged for Xbox and Microsoft Windows in June 2005, at that point discharged on Xbox Originals for Xbox 360 in December 2008 and after that discharged for Playstation Store and PSN for PS3 in December 2012.

As indicated by the 2008 deals appraisals, it has been pronounced to be the smash hit round ever on PlayStation 2. It was accessible in Steam stage by January 2008 and on Mac OS X10.6.6 by September 2011. Grand Theft Auto: San Andreas was discharged for Playstation 3 by means of Playstation Network by December 2012. San Andreas is organized like the past two variants. The gameplay comprises of components of a third-individual shooter and a driving amusement, permitting the player an open world condition in which to move unreservedly. The player character can walk, eat, run, dash, swim and move and in addition hop and utilize weapons and different types of hand to hand battle, all by walking. As opposed to taking them player can import vehicles of autos, transports, semis, pontoons, settled winged flying machine, helicopters, trains, tanks, cruisers and bicycles.

When one takes into consideration the storyline of GTA: San Andreas, as Murray notes, is basic [7]: after the demise of his brother five years ago, Carl Johnson returns back upon the death of his mother. However, this time, he is accused of murdering a cop as well. In the meantime, the situation of CJ's 
neighborhood and his surviving family is disarrayed due to destructive impact of weapons, medications and skewed law implementation. What the player does is to lead CJ through missions that emerge from adolescence hanging-out spaces to urban communities in order to control his neighborhood again. However, in this version, $\mathrm{CJ}$ is not a criminal from the start [7]. As Murray explains, in GTA III, the main hero is a gotten away convict out for vindicate; remaining anonymous, he is alluded to with the second-personal pronoun "you" and not being an unspeaking hooligan upon whom we can portrait a variety of characteristics, our hero is Tommy Vercetti, an Italian-American intense person reminiscent of the HBO series The Sopranos with cinematic allusions of Scarface [7].

According to Murray, the designer of the game imitated 1990s style hood films like Colors (1988), Boyz N the Hood (1991), and Menace II Society (1993), with one of the co-authors of San Andreas being DJ Pooh, screenwriter for the independent ghetto dramafilm Friday (1995) [7]. If it could be reminded, a $\mathrm{Cu}$ ban immigrant named Tony Montana as the movie's protagonist was creating a fortune through narcotics. As noted by Murray, in similarity, Vice City's Tommy Vercetti also creates an economic rise by any means necessary and thus by taking advantage of unmistakable cultural signifiers like film, fashion, music and the vulgar language, the creators of GTA are able to establish a virtual sense of "place" that enhances one's general understanding by making a more consistent condition in which to establish the act of CJ [7].

The game viably dives the player into the hazardous world CJ must explore, through its unyielding materiality. While this may appear to be contradictory to a condition that exists just on an enlightened screen and in the psychological topographies of its players, nevertheless, GTA passes on a discernable urban surface furthermore, enunciated feeling of body involvement. San Andreas presents an immense topography containing eight separate areas and regions. Each locale inside the condition of San Andreas has its own particular unmistakable feel and essentialness. Los Santos feels particularly like Los Angeles with its dirty heaven skies, palm trees, and electrical cables, its grime and sparkle all cluttered together. Rock County, Whetstone, and Red County are emphatically provincial, crosscut with dusty streets and once-over towns that give locales for grimy dealings best kept past as far as possible. San Fierro, reminiscent of San Francisco, is arranged as the most diverse zone with a refined feel and a brilliant grouping of elective subjects. Rural segments are all Technicolor blue skies and prepared gardens, wide boulevards, and less recognizable streetwalkers. Extensions and underpasses isolate areas, making it is important to build up an ability to read a compass and find effective approaches to move about the city. Players figure out how to explore the dirty urban sprawl by perceiving historic points and recollecting alternate routes. Truth be told, accomplishment in the diversion is to a great extent dependent upon viably arranging and remembering the virtual territory.

Fleetingness orders the reenacted spaces of Grand Theft Auto too, and this is 
absolutely one of its best procedures as far as rendering a persuading condition [5]. It exists, however in truncated shape: one moment of genuine time meets one moment; one moment rises to one hour and twenty-four minutes turns into a day. Amid these every day cycles the sun rises and sets, as does the moon. There are seasons and climate designs. As the sun plummets over GTA's most recent region, San Andreas, the "focal point" that speaks to the player's perspective flares from its glare. In an overwhelming deluge, it turns out to be harder to control one's vehicle. Perceivability contrasts in view of the nature of the day. At sunset, the city lights flash on. Movement winds up plainly thick amid surge hour, and in the early morning hours it is less crowded. Planned missions require an insightful feeling of both the time and exact area of one's goal, and without these things advance cannot be made in the game. In like manner, players build up an ability to read a compass through rehashed route of a consistently expanding domain, and will probably know the game space-the virtual city-superior to anything they know as their own "genuine" neighborhoods. A fascinating new worldview of virtual open space develops when one considers becoming lost in a city that exists just in the time and space of the mimic.

The game's powerful interface between the player's body and on-screen character is accomplished through a muddled arrangement of relations that depend on faculties of touch, sight, and sound. In San Andreas, for instance, a persuading sense regarding one's character-body is created through stylish criticism. The game's interface, a hand-held simple controller, vibrates stunning when CJ pitches down a lofty, rough slope on a soil bicycle. With expanded speed, the obscured visual picture seems to shake, just as recorded by a hand-held camera. The joined impact is that of a wild ride on a vehicle that is scarcely under control. What's more, however the bobbling picture and thundering controller not the slightest bit duplicates the "genuine" feeling of diving downhill on a BMX bicycle, the visual dialect successfully passes on the elating surge in to a great extent true to life terms. The expanded modernity of rendering takes into account more practical communication with the reenacted condition: slip marks show up when an auto peels out and about, an easy route through free earth sends a shower of mud along the flanks of one's vehicle. With the unavoidable impact, one's auto mirrors that harm with imprints, smashed windows, and lost guards. A punctured tire sends flashes flying from the wheel well and renders the vehicle crippled. Lowriders with water power ride contrastingly out and about than sports cars, which thus handle particularly from light trucks or vans.

In spite of the fact that the storyline missions are important to advance keeping in mind the end goal to open certain urban areas and other substance, the open-world, non-direct condition permits players to investigate and pick how they wish to play the diversion. While not finishing one of the storyline missions, the player can free-meander and look around the city, eat from the eatery or cause tumult by assaulting individuals or cause devastation. Making destruction will draw in undesirable consideration from the experts of police, FIB, SWAT 
groups or even military. Single missions, for example, dropping off taxi travelers, putting out flames, driving harmed individuals to doctor's facility and battling a wrongdoing as a vigilante are all incorporated into this form also. Be that as it may, there are new extra side-missions for example, thievery, pimping, truck and prepare driving missions which require players to make conveyances on time, driving or biking to schools. These missions help the player to learn aptitudes and systems to use in the comparing vehicles.

A similar feedback relationship is developed with compositional structures also, with San Andreas, another level of instinct and course is possible in an arrangement of clothing stores, barbershops, rec. centres, ammunition shops, bars, and different business establishments. In antagonistic region, match posses shred your vehicle with gunfire. Ganton, the saint's home turf, is beaten and has the pathetic, slight, hanging appearance of a crime-perplexed, disregarded place. Remarkably, the authorities from time to time meander onto this piece, however the police presence in rich locales is more evident and compelling. Through the kept up control and control of various unmistakable territories, one quickly sees on a very basic level the carceral nature of the entire region of San Andreas. Over the traverse of game play, issues of building accessibility and limit, the moving use-estimation of a site-furthermore comes into question. For the version shows in the meantime both an assortment of prominent designing edges (strip malls, stadiums, handling plants, air terminals), and after that those to a great degree same structures made irregular through their change into heterogeneous areas of culpability and degradation.

Territories, for instance, "pay'n'spray", practice center and shops twist up perceptibly open to the player basically after the realization of particular missions. In case that the player were to go in the catapulted zones, they would get a six-star required level. For example, in a manner of speaking, Los Santos is available to the player however opening diverse urban zones and commonplace locales require the realization of particular missions. Notwithstanding the way that soaking inside the diversion space depends on GTA's striking aural condition, sound remains a significantly understudied segment of electronic entertainments. Despite the incalculable parts like development commotions, vociferous citygoers and unmistakable huge name voiceovers, radio incitement gives an energetic soundscape. In San Andreas, as in the earlier phases of the diversion, hit records from the appropriate day and age orchestrate the player into a true blue request. The gangsta rap from the mid nineties that impacts from the autos CJ drives fuses NWA, Dr. Dre, Eazy E, and Tupac, each one of whose voice the issues of police severity, the inability to find true work, and the fierceness of ghetto life. Every now and again frightfully misconstrued, the quality of these pros' verses abides not in what intellectuals see as a celebration of exacting brutality, realism and besides, misogyny. Rather than pushing gangsterism, rap verses and the hip-jump attitude advocates the right hardening of will and change of the forcefulness imperative to shed camouflaged opinions of victim- 
hood to get a handle on grandstand competition.

San Andreas' story goes up against another criticality through these songs, as one is constrained to investigate the traps of the Idlewood (a.k.a. Inglewood) and Ganton (a.k.a. Compton) ghettoes [6]. Through this sound, the version winds up obviously recorded with a kind of care that fills in as an antithesis to the clear political oversight of the visual space. What rises is the version's subtext: an affair of the dystopic city, punctuated by a sharp unexpected comment on the certain debasement and false respect that would direct such a reality. With the extra layers of self criticality that drive the storyline, $\mathrm{CJ}$ is reconfigured not as just a horrendous, bodily hoodlum with Crafty ways, however a kind of normal business visionary who is constrained to exist outside the framework. His story twists up discernibly one of making sense of how to circumnavigate the unpleasant condition and ascend in a World in which he ought to intentionally move between both the law and the insubordinate.

The most enormous qualification from the GTA III and GTA: Vice City, GTA: San Andreas does not require a stacking screen when the player is in movement [7]. The primary stacking screens in the game are for cut-scenes and internal parts. Distinctive differences join the change from single-player to multi-player missions and the substitution of the "concealed bundles" with covered camera shots, horseshoes and shellfish to discover.

In like manner, without precedent for the series, the player can swim and climb dividers. Since water is never again a shut prevention, the ability to swim incredibly influences the player. Despite the refinements is for more important ability, the player can utilize twofold firearms or play out a drive-by shooting with various posse individuals.

Posse wars are unmistakable. At whatever point the player meanders into enemy area and murders no under three group individuals, battles with foe groups start. The player will at first take control of the areas of distinctive groups to shape his/her own. At whatever point her/his own specific group is framed, its area will be attacked too so it will be critical to hold the picked up domains. Once the player takes control of the considerable number of districts, none can go under attack.

Are games any less a space of execution and play since they do not include "lived" circumstances? With Grand Theft Auto: San Andreas, a player is tried and true to an extended degree for a reproduced body that requires typical support and work out, and that can be dressed and adorned with tattoos and haircuts. This is a body that developments with evolving shocks, for instance, a sound versus trash support abstain from food. (It winds up detectably colossal to observe that in CJ's neighborhood, as various underserved interior city goes, the principle three diners around the neighborhood fast sustenance: burgers, fricasseed chicken, or pizza.) This is a body that modifies in perspective of oxygen devouring versus quality building endeavors, whose driving aptitudes and drawing in quality to the backwards sex increase with experience, and whose 
muscles swell with work out, or whose stomach swells with glutting. More sensible than it may show up, a player controls qualities like respectability, fat, muscle, stamina, and sex offer as demonstrated by the necessities of each mission. With too much fat and lacking stamina, a loping Carl cannot "physically" remain mindful of his objectives. By taking a shot at holding his breath while swimming submerged, he continuously improves his virtual lung restrict. Carl's diverse woman companions also put requests on him in regards to keeping his body the way that they like him: a couple of them like him slender, others are slanted toward a more liberal form. A hot car gives him a higher sex claim rating; showing up at his sweetheart's home with that same car stamped cuts it down. As the diverse headways of the game turn out to be ceaselessly puzzling, the virtual body of the hero seems to ask progressively of what our genuine bodies require, while satirically replicating the social weights related with shallow appearances and realism.

Not exactly the same as the previous series, there is the property of robbery as home interruption, which is consolidated as a potential for a money making activity. By taking a robbery van, the player can sneak into a living arrangement amid the night and take the resources.

Not exactly the same as the past versions, in GTA: San Andreas, different mini-games are open. These are ball, pool, rhythm-based troubles and arcade games.

The player can spend his/her exchange out in betting, tattoos, dinners et cetera. Outrageous betting misfortune can compel the player to sink into commitment, which shows up in red and negative numbers.

To frustrate matters, this reproduced substance that solicitates perpetual keenness with respect to his physicality is especially an underprivileged, urban, African-American male. With San Andreas, RockStar has taken the poor dull male body, which is encoded as a human stain on the surface of a squeaky-clean American longing for opportunity, and pushed it into the point of convergence of our thought. This debased presence constitutes a sign of a contemptible history of genocide and subjugation. Ideologically organized as base, grinning, dirty, detained, and exhausted, the dim body is the extra of a national condition; a glitch that cannot be consumed into the system. Be that as it might, now, that signifier of the dull body, that shell whereupon such a vast number of negative affiliations has been expected, transforms into a mirror for a thorny gathering of societal relations in America.

There is the property of multi-player. In addition, there are multi-player furors in which both of the players are shown at the same time on the screen as they ought to stay in closeness to each other.

Meanwhile, Rockstar encourages us to recollect how fetishized that body truly is or, more decisively, will advance toward getting to be plainly resulting to the social depiction of the game. For the moment in which this game is organized lies as of late past to the "bling-bling" period in which hip-jump transformed 
into a rich undertaking and began to have an overall group influence. The rap music leaving the time duplicated in this game, as it were, addressed a break with Civil Rights period techniques for protection and social raise. Rather than contingent on assimilation into the standard, a young hip-bounce period began to compellingly get a handle on free endeavor and extreme autonomy as strategies for accomplishing the American dream. Monetary impact advanced toward getting to be the new course to social and political effect. In this, as well, with CJ: burglarized of his cash toward the start of the game and under the huge weight of a social system that was not proposed for his favorable position, he, in any case finds ways and means. It is the outrageous achievement of hip-bounce, addressed in its flourishing structure in San Andreas, which prefigures the bona fide credibility of a character like $\mathrm{CJ}$ including the central hero's part in such a game. It shows up a startling reversal of the Hollywood model, in which the shared characteristic of the principle parts is overwhelmingly portrayed by whiteness or racial dubiousness. Rockstar's capacity to put Carl Johnson in advance in its now awesome GTA arrangement presents affirmation that, in a \$23 billion electronic gaming industry, it is in actuality workable for a dull, male saint to have mass intrigue. The circularity of the redirection's searing message works feasibly both inside and outside the limits of the medium, revealed through a player's significant engagement with the automated geographies of San Andreas.

Electronic games re-indicate "living" spaces in which a modernized delegate works together with various administrators, giving sorting out grounds whereupon to play. In this imagined space, the normal social contract is suspended; in any case, this is not to express that what comes to fruition is a boorish space. Or on the other hand such games as Grand Theft Auto address administer-based, basic reasoning conditions that require creative courses of action inside a described arrangement of parameters. Thinking about the estimation of a virtual city as both a circumstance in which architects may re-show trusts and sentiments of fear with respect to societal issues and a place where it is workable for a player to play out his/her relationship to these contemplations in a not-precisely constituted site is a liminal experience that shifts in the nonexistent and in philosophy.

The topographies that I have portrayed are imagined and envisioned, no uncertainty. In any occasion, games are a light distraction; at most, they constitute nuanced areas for an examination of another kind of liminal reality. Perhaps games are only the more canny variation of existing filmic conventions, anticipated that would toy with classy experience of a greater extent game purposes. For me, these depictions of urban spaces can fill in as objective zones in which to indicate more unavoidable (and all the more "real") pressures around lived social conditions. Opportunity City, Vice City, the streets of the staggeringly expansive territory of San Andreas-these are all cutoff zones in which it winds up perceptibly possible to test safely with enormously stupefying parts of present 
day life. A world-creation mimetic edge, electronic gaming is one social impression of the vertigo related with the puzzling stream of overall economies and the colossal weight on the individual to alter and get by inside it. Notwithstanding different things, Grand Theft Auto's geologies pushed toward getting to be stages whereupon to bear on techniques for installment for the unprecedented uncertainty of position (subject position, cash related status, simplicity of character) related with current life.

Another system that was utilized as a part of this variant was about the nature of particles [2]. Assessing Particles Quality is one of the trickier undertakings in this guide-the look of blasts are randomized, material science and climate frameworks cause extra variety and the delayed consequences adjust as well. What we can state is that as the setting is raised blasts and different impacts increase expanded volume and detail and when set to High or Very High, molecule shadows are rendered as well.

The other technique that is utilized is the Reflection Quality [2]. Reflection Quality modifies the lucidity of the reflections found on vehicles, sparkling floors, Windows, puddles, open waterways and the incidental mirror, similar to the ones in bedrooms and hairstyling salons.

Clearer is the distinction between Very High and High, which sees the precision of reflections additionally diminished. On Normal Level, reflections lose all detail and reflections on different surfaces are completely evacuated, something you will see quickly in the second arrangement of examinations.

Out in the open World, the contrasts between the Reflection Quality subtle elements are more unmistakable: Ultra to Very High outcomes in a softening of bigger reflections, Very High to High further diminishes lucidity and High to Normal sees reflections expelled from each vehicle, window and building. Besides, the few outstanding reflections found on waterways and intelligent floors experience the ill effects of artifacting, gleaming more than once.

The most recognizable effect of this procedure is in hair saloons and homes, where top notch mirrors can be seen very close and the encompassing intelligent detail is impeccably duplicated instead of approximated.

\section{Version 6-Grand Theft Auto IV (2008)}

Following a six months-delay, this version was released on 29 April, 2008. It is the primary form to be released both for Sony and Microsoft's PC game consoles.

The game occurs in a refreshed Liberty City that resembles New York City, generously more than the past adaptations. This version formally introduced online multiplayer with the series. The game does not offer split screen or LAN multiplayer modes on Playstation 3 or Xbox 360 however there is LAN on the PC mode. Up to 16 (32 on PC) players can play together completing a grouping of games including Death Match, Deal Breaker and Mafiya Work et cetera.

Grand Theft Auto consolidates online multi-player with 15 techniques for play accessible. It supports up to 16 players and empowers them to research the 
city. Hosts of the game can control by far most of the elements, for instance, police presence, traffic and weapons.

The online games are separated into situated and unranked matches. The reward for the positioned gameplay is cash which chooses players' positions. Players use a versatile character in the greater part of the play modes and cash earned in the midst of play a little bit at a time makes more customization choices open.

A couple of particular game modes are accessible. Group based gameplay modes fuse Team Deathmatch in which 2 - 8 groups battle to gather the most butchers in a standard deathmatch; Team Mafiya Work, in which 2 - 8 gatherings battle to complete contract work for the "Mafiya"; Team Car Jack City where 2 - 8 bunches battle to take cars and get money for keeping undamaged and Turf War including two gatherings who battle to take control of doled out scopes of the guide and control them for whatever period of time as long as possible.

The game in like manner fuses a grouping of hustling and supportive modes for the multiplayer modes which are Race, in which players race through checkpoints in a regular vehicle race; Hangman's N.O.O.S.E., an inside mode that expects players to assemble a man from the air terminal and safely escort him to the extraction point before cops kill him; Deal Breaker, a center mission that expects players to snare an development site got by enemies seek after a social affair of foes before they escape, a Free Mode in which players have the entire outline to explore with no extreme target or mission to wrap up [8].

Gunfights in GTA IV are coordinated by using a third-individual framework. The game empowers the player to move between covers, to fire unpredictably, to point transparently and focus on a specific foe. Particular body parts can in like manner be centered around.

Niko's prosperity shows up by a green sickle on the left $50 \%$ of the mini-guide while a blue semi-float on the benefit addresses protective layer. On the off chance that Niko's is hurt, he can recover prosperity by eating, drinking, resting or using restorative packs. In case Niko's prosperity accomplishes zero, the action stops and he re-appears at the nearest specialist's office having lost some money yet holding his weapons.

The Wanted Level has changed in this version. Regardless of the way that the starting levels show up with a comparable measure of stars using a comparative plan, the law approval workplaces which may look the player have changed with the center enthusiasm on making them more sensible. In GTA IV, the police are helped by NOOSE (National Office of Security Enforcement) officers at a three-star required level which are joined by the Tactical Response Unit or FIB (a parody of FBI) at larger amounts. Outfitted power is not open in this variation. Like the past GTA adaptations, a police helicopter looks after the player at a three-star required level yet this is supplanted by a helicopter gunship at the five-star level.

Basic to all variations, including GTA IV, vehicles give the extraordinary 
techniques for movement. Every vehicle in the game uses a mini-guide and a GPS contraption. In this version, the player can in like manner hail a taxi so going between objectives without driving winds up is perceptibly possible. The journey might be skipped additionally with the objective that the player can arrive at the goal rapidly. In the midst of auto pursues, the player can focus the camera on the target vehicle by grasping the practical camera catch and moreover free-call attention to discharge out of the vehicle using one-handed weapons.

Not the same as the past adaptations, GTA IV uses "Ace Wireless" flexible phones as opposed to open telephones to hand-off missions to the player. It has a couple of utilities like text messages and appointments, sorting out to meet associates for practices and retrying failed missions. The player can in like manner take photos for particular missions or call 911 to summon emergency calls. The phone too empowers access to the amusement's multiplayer mode. The player can call different characters to chat with them or demand an organization they can give, for instance, transportation or a diminishment in the required level.

One of the techniques that was presented inside this rendition was the Quality of Shader [2]. Shader Quality influences the devotion of lightning and lightning impacts cast on surfaces, however in Grand Theft Auto V extra employments of this technique are likewise noted.

Around evening time, minimizing to Shader Normal outcomes in the loss of sprout and gleam from splendid lights, diminishing constancy at the dock and all through the World.

The second procedure that was introduced with this adaptation was the Quality of Shadows [2]. Shadow Quality Works as an inseparable unit with High Resolution Shadows to enhance the definition and exactness of all shadows, as in Distance Scaling and Extended Distance Scaling coordinate to enhance level of detail.

Notwithstanding the undeniable increment in shadow devotion, take note of the exactness of the shadows on the houses. As Shadow Quality is diminished, the general exactness of shadowing diminishes, bringing about the inevitable loss of shadows under the shades of housetops. Thus, the detail and exactness of shadows under, close by different surfaces is incredibly diminished.

Besides, the utilization of High outcomes in the loss of vehicle and NPC shadows cast by vehicle headlights and on Normal shadows cast by foliage and different products are likewise lost, fundamentally affecting picture quality.

\section{Version 7-Grand Theft Auto V (2013)}

This version was released on 17 September 2013 for the PlayStation 3 and Xbox 360 consoles. It is the fifteenth title in the Grand Theft Auto series, and the first essential entry since Grand Theft Auto IV in 2008. As one of the last titles to be released for the seventh time of PC game consoles, Grand Theft Auto V has been exceptionally anticipated going before its release.

Grand Theft Auto V is an activity experience PC game played from a 
third-individual perspective in an open world condition [9]. The player uses conflict strikes, firearms and explosives to battle adversaries, and can run, bob, swim, or use vehicles to investigate the game's world. In fight, car point and a cover framework can be used as help against adversaries. In case the player's prosperity is low, it will a little bit at a time recuperate to its midpoint. A ceaseless target for the player is to complete missions to progress through the story, however much like other open world recreations, these missions can be done at the player's unwinding. If the player submits unlawful acts while playing, the game's law prerequisite associations may respond, which is addressed by a "Wanted" meter in the head-up display (HUD). On the meter, stars are appeared to exhibit the player's available "Wanted" level; for example, and no more outrageous five-star "Wanted" level the undertakings by law necessity to debilitate the player end up being astoundingly intense. Should the player make tracks in an opposite direction from the incite area in which they are required, law necessity officers will search for the player. Presently, the perceptible pathway of officers appears on the small guide; when distinguishable pathway is broken and the player is covered, the "Needed" meter enters a chill off mode and at last dies down.

The single-player method of Grand Theft Auto V is played through three player-controlled heroes: Michael, Trevor, and Franklin. They are guilty parties whose stories interconnect as they complete missions. While participating with the entertainment world, the player may switch between them willfully, by strategies for a directional compass on the HUD. Franklin relates toward the north direct, Michael toward the west, Trevor toward the east, and the player's multiplayer image toward the south. In the midst of the missions, the amusement may switch the player's character normally as basic remembering the ultimate objective to complete certain objectives. A character's image will streak red if they are in hazard and need assistance either physically or through covering fire from another, and streak white if that character has a key favored point of view or position which may help the player's achievement in the mission. Despite the way that the player completes missions as any of the three legends, the more troublesome heist missions may require assistance from AI-controlled partners who have extraordinary capacity sets, for instance, PC hacking or driving, and they will take a cut from the mission's cash compensation upon fulfillment. In case an embellishment survives a productive heist, they may be re-used as a piece of later missions with moves up to their unique aptitudes. Using unmistakable strategies toward the completing of a heist mission is furthermore enabled by the game; for example, in a robbery mission the player may quell normal people with an administrator if stealth is upheld, or tempest the scene with guns drawn.

Another technique that has started to be utilized beginning from this rendition is DirectX rendering [2]. Fantastic Theft Auto V offers players the decision of three DirectX renderers: DirectX 10, DirectX 10.1 and DirectX 11. DirectX 10 
and DirectX 10.1 are incorporated basically for similarity purposes, empowering those with more established GPUs lacking DirectX 11 support to play the game. Be that as it may, it may be thought about whether they are any quicker, in spite of DirectX 11 by and large being better improved and having a predominant list of capabilities and DirectX 11 game bolster being as often as possible calibrated through normal GeForce driver discharges. Obviously, benchmarks uncover that prevalent exhibitions are accomplished when utilizing DirectX 11.

The second technique presented in the last version is tessellation [2]. Tessellation all in all adds additional geometric detail to surfaces, objects and characters. In May Payne 3, the last RockStar RAGE Engine game to be discharged on PC, tessellation was utilized nuance to add bend to Max's ear and garments and to the tires of vehicles. In Grand Theft Auto V, its utilization is similarly unobtrusive, adding subtle element to choose trees, the intermittent bramble, a few links and a few waterways.

Of these improvements just tree tessellation is observable amid gameplay and is moreover the main component sufficiently obvious to catch in screenshots.

As the detail is raised, the measure of tessellation added at each progression declines to the point where Very High's change is near on difficult to spot. It is around the base and center of the right-most tree in the forefront and close to the base of the tree before the ball court.

The last technique that was presented with this last version was about the Quality of Texture [2]. With Parallax Occlusion Mapping fixing to Shader Quality and different methods of note isolated, there are no curve balls while analyzing Texture Quality.

High is generally indistinguishable to Very High at ground level, however now and again Very High's surfaces will be more honed when seen in first individual, facilitate from the floor some bigger and less vital surfaces are of a lower quality to marginally decrease VRAM utilization. In general however the distinction is not articulated. On Normal Level, be that as it may, everything endures a sizeable shot, diminishing the devotion of each finished game component, which incorporates the surfaces connected to water surfaces.

\section{Conclusions}

GTA IV-as a game about socialization-advises its group of onlookers of the key to progress, the best approach to obtain the desires of society, and the belief systems that must be received as a method for making due in the "concrete jungle". The methods, pleasantly expressed above, are to break free of institutionalized standards and grasp humankind's normal condition that appropriates the American Dream through criminal development. With this understanding, I discover the implications behind the savage demonstrations in GTA's all versions to be significantly more harmful than the potential impacts created just as a reaction to visual boosts. For example, as opposed to simply painting the lanes of Liberty City with violence, GTA IVs story uncovered the inspirations that lead 
numerous visuals to dismiss societal standards and wander down a way to wrongdoing.

Extraordinary designs do not simply occur overnight-they set aside time to get right. UX originators and, actually, all different sorts of creator realize that they do not get one attempt to make a product as well as can expected be, it takes numerous cycles to get the item the way that it best suits the user. Iterative design forms are utilized over all design orders to deliver outcomes about that count.

The iterative design process is a straightforward idea. This iterative design is frequently called "rapid prototyping" or "spiral prototyping". Once, through user research, you have recognized a user need and have produced thoughts to address that issue, you build up a model. At that point, you test the model to see whether it addresses the issue in the most ideal way. At that point, you take what you gained from testing and revise the design. Following that, you make another model and start the procedure once more until the point that you are fulfilled to have achieved the most ideal product for discharging to the market.

Because of this thought and with the examinations done for every adaptation all through this article, one could state that all through the distinctive variants of GTA, it could be anticipated that its generation is that of a tedious procedure. With every form, new highlights and strategies have been included and executed. What is more, it additionally ought to be included that each past version has served a prototype for the coming form and hence a repetitive procedure of cycle has been utilized as a part of the production design of Grand Theft Auto.

One could contend that every single version of Grand Theft Auto has been actualized with new procedures. Before every version was released out into the market, a model has been created to see whether it addresses the issues of purchasers and in like manner the new form has just been released a while later with new highlights included. In this manner all things considered, one could state that through an interactive design process, the whole advancement of Grand Theft Auto as an adventurous game with the most popularity in the most recent decade has survived.

\section{References}

[1] Beck, B. (2009) Something for the Boys: Iron Man, Transformers, and Grand Theft Auto IV. Multicultural Perspectives, 11, 27-30. https://doi.org/10.1080/15210960902717353

[2] Burnes, A. (2015) Grand Theft Auto V PC Graphics \& Performance Guide. GeForce, NVIDIA.

https://www.geforce.com/whats-new/guides/grand-theft-auto-v-pc-graphics-and-pe rformance-guide

[3] Osborne, P. (2011) Evaluating the Presence of Social Strain in Rockstar Games' Grand Theft Auto IV. Studies in Popular Culture, 34, 109-132.

[4] Miller, K. (2008) Grove Street Grimm: Grand Theft Auto and Digital Folklore. The Journal of American Folklore, 121, 255-285. https://doi.org/10.1353/jaf.0.0017 
[5] Salter, M.B. (2011) The Geographical Imaginations of Video Games: Diplomacy, Civilization, America's Army and Grand Theft Auto IV. Geopolitics, 16, 359-388. https://doi.org/10.1080/14650045.2010.538875

[6] Miller, K. (2007) Jacking the Dial: Radio, Race and Place in "Grand Theft Auto". Ethnomusicology, 51, 402-438.

[7] Murray, S. (2005) High Art/Low Life: The Art of Playing “Grand Theft Auto". PAJ: A Journal of Performance and Art, 27, 91-98. https://doi.org/10.1162/1520281053850866

[8] Barrett, P. (2006) White Thumbs, Black Bodies: Race, Violence, and Neoliberal Fantasies in Grand Theft Auto: San Andreas. Review of Education, Pedagogy, and Cultural Studies, 28, 95-119. https://doi.org/10.1080/10714410600552902

[9] Walton, M. and Pallitt, N. (2012) “Grand Theft South Africa”: Games, Literacy and Inequality in Consumer Childhoods. Language and Education, 26, 347-361. https://doi.org/10.1080/09500782.2012.691516 